\title{
Inhibition of P65 and PDK2 Increases Cytotoxicity and Radiation Sensitivity of Pancreatic Cancer Cells
}

\author{
Joshua A. Streveler ${ }^{1,2}$, Helen Chin-Sinex ${ }^{1,2}$, and Marc S. Mendonca ${ }^{1,2,3}$ \\ ${ }^{1}$ Indiana University School of Medicine, ${ }^{2}$ Department of Radiation Oncology, \\ ${ }^{3}$ Department of Medical and Molecular Genetics \\ Indiana University School of Medicine, Indianapolis, IN 46202
}

Background: Pancreatic ductal adenocarcinoma (PDAC) is currently the $4^{\text {th }}$ leading cause of cancer death in the US because of late detection and resistance to chemotherapy and radiation. This tumor resistance is partially due to high activity of the transcription factor, NF- $\kappa \mathrm{B}$ which promotes cell survival and pyruvate dehydrogenase kinase 2 (PDK2) which regulates aerobic glycolysis (Warburg Metabolism), cell proliferation, and inhibition of apoptosis. We have shown that simultaneous treatment with the chemical NF-кB inhibitor DMAPT and the Warburg inhibitor DCA is cytotoxic and enhances radiation-induced cell death in human PDAC cells. We have also shown that double gene knockdown of the P65 (RELA) subunit of NF-kB and PDK2 by siRNA increased cytotoxicity and radiation sensitivity of pancreatic cancer cells but to a lesser extent than DMAPT and DCA treatment.

Experimental Design: Western blots suggested the siRNA did not completely suppress $\mathrm{p} 65$ and PDKS protein levels. To test the hypothesis that more complete inhibition of NF-kB and PDK2 is required to increase cytotoxicity in PDAC cells, we transfected the Mia $\mathrm{PaCa}-2$ cell line with appropriate siRNA and exposed the cells to the chemical inhibitors DMAPT and DCA. The cells were plated and followed by irradiation with either $0,2,4$, or 6 Gy of $160 \mathrm{kVp}$ X-rays.

Results: Compared to previous experiments that utilized siRNA knockdown alone, the combination of dual drugs and dual siRNA shows an increase in both cytotoxicity and radiation-induced cell killing ( $p<0.001, t$ test).

Conclusion: The data suggest that a more definitive approach such as utilizing CRISPR-Cas9 may be required to ensure complete gene knockdown of p65 and PDK2 to elucidate the mechanism(s) by which simultaneous inhibition of NF-kB and PDK2 inhibition enhance cytotoxicity and radiation-induced cell killing in pancreatic cancer. 